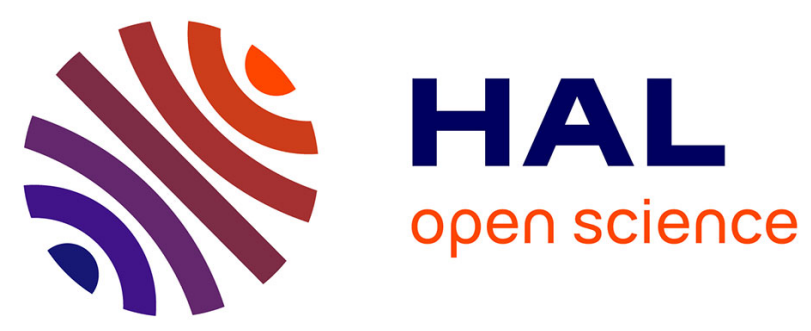

\title{
Transferts de technologie et accumulation de capacités technologiques dans les entreprises d'Etat de l'industrie électronique en R.P. de Chine (1978-1991)
}

Jean-François Huchet

\section{- To cite this version: \\ Jean-François Huchet. Transferts de technologie et accumulation de capacités technologiques dans les entreprises d'Etat de l'industrie électronique en R.P. de Chine (1978-1991). Revue Tiers Monde, 1995, 36 (143), pp.661-684. 10.3406/tiers.1995.4985 . hal-02539752}

HAL Id: hal-02539752

https://hal.science/hal-02539752

Submitted on 10 Apr 2020

HAL is a multi-disciplinary open access archive for the deposit and dissemination of scientific research documents, whether they are published or not. The documents may come from teaching and research institutions in France or abroad, or from public or private research centers.
L'archive ouverte pluridisciplinaire HAL, est destinée au dépôt et à la diffusion de documents scientifiques de niveau recherche, publiés ou non, émanant des établissements d'enseignement et de recherche français ou étrangers, des laboratoires publics ou privés. 


\section{Transferts de technologie et accumulation de capacités} technologiques dans les entreprises d'État de l'industrie électronique en République populaire de Chine (1978-1991) Jean-François Huchet

\section{Citer ce document / Cite this document :}

Huchet Jean-François. Transferts de technologie et accumulation de capacités technologiques dans les entreprises d'État de I'industrie électronique en République populaire de Chine (1978-1991). In: Tiers-Monde, tome 36, n¹43, 1995. Professions scientifiques en crise. Ingénieurs et médecins en Syrie, Égypte, Algérie. pp. 661-684;

doi : https://doi.org/10.3406/tiers.1995.4985

https://www.persee.fr/doc/tiers_0040-7356_1995_num_36_143_4985

Fichier pdf généré le 30/03/2018 


\title{
TRANSFERTS DE TECHNOLOGIE ET ACCUMULATION DE CAPACITÉS TECHNOLOGIQUES DANS LES ENTREPRISES D'ÉTAT DE L'INDUSTRIE ÉLECTRONIQUE EN RP DE CHINE (1978-1991) ${ }^{1}$
}

\author{
par Jean-François HuCHET ${ }^{2}$
}

\begin{abstract}
Après une longue période de fermeture durant laquelle la Chine a incarné auprès des pays en voie de développement (PVD) une politique de développement industriel autocentrée, la fin des années 1970 marque un revirement important avec un recours systématique aux technologies étrangères. Dans l'industrie électronique, on estime qu'environ les deux tiers des entreprises d'Etat auraient importé une technologie durant la période 1978-1990'. Durant les $\mathrm{VI}^{\mathrm{e}}$ (1980-1985) et VII ${ }^{c}$ Plan (1986-1990), le montant des dépenses engagées serait de l'ordre de 3 milliards de dollars us, répartis sur un peu plus de 2000 contrats (R. Conroy, 1990 ; D. F. Simon, 1991); à cela, il faut ajouter le recours aux investissements directs étrangers (IDE) qui, à partir du milieu des années 1980, se développe très rapidement, faisant des régions du littoral chinois un des principaux récipiendaires de capitaux étrangers dans le monde.

Le transfert international de technologie constitue un des principaux moyens par lequel les entreprises des anciennes économies socialistes vont pouvoir s'insérer dans des industries mondiales où les capacités d'innovation sont de plus en plus déterminantes dans la compétitivité des firmes. De ce point de

1. Cette recherche est issue d'un long sejjour de recherche en RP de Chine entre 1987 et 1991 pendant lequel j'ai effectué une enquête à partir d'un questionnaire diffusé dans près de 20 entreprises d'Etat et coentreprises dans le secteur électronique, bénéficié de l'accès à des recherches universitaires locales sur le résultat de la politique de transfert de technologie portant sur plus d'une centaine d'entreprises d'Etat, et réalisé de nombreuses interviews auprès des ofliciels chinois en charge de la politique de transfert de technologie et de l'innovation ainsi que des responsables d'entreprises étrangères ayant réalisé des transferts de technologie dans l'industrie électronique chinoise.

2. Pensionnaire-chercheur à la Maison franco-japonaise, Tokyo.

3. Zhonguo Dianzi Nianjian. 1986 et 1991 (Annuaire de l'industrie électronique chinoise).
\end{abstract}


vue, la Chine est entourée de voisins vertueux qui, comme le Japon depuis plus d'un siècle, ou les Nouveaux Pays industriels (NPI) depuis les années 1960, ont su tirer parti avec une redoutable efficacité d'un apport de technologies étrangères, alimentant ainsi les thèses d'un modèle asiatique en matière de rattrapage technologique. La Chine est elle en train de suivre cette voie ? Offre-t-elle un exemple auprès des anciennes économies socialistes en matière de transition technologique?

Cette vague d'importation massive de technologies depuis 1978 n'est assurément pas étrangère au développement exponentiel de la production électronique nationale et au positionnement compétitif sur plusieurs segments concurrentiels des marchés internationaux (1). Cependant, ces résultats spectaculaires cachent des situations différentes sur le plan de l'efficacité dans l'appropriation des technologies étrangères. Dans le cadre des contraintes imposées par la globalisation croissante de l'industrie électronique sur les chances de rattrapage technologique pour les PVD, notre recherche fait apparaître des capacités d'appropriation différenciées selon les entreprises chinoises : ces capacités sont en grande partie liées aux droits de propriété qui façonnent l'environnement dans lequel se réalise l'effort technologique des entreprises. Le bilan sur près de quinze années de politique d'importation de technologie par les entreprises d'Etat dans l'industrie électronique fait apparaître de piètres résultats par rapport aux objectifs annoncés au moment de la politique d'ouverture en 1978 (2) et contrastent avec ceux plus prometteurs enregistrés dans les investissements directs étrangers'. La poursuite d'une régulation bureaucratique de l'entreprise ainsi que des déficiences dans la gestion macro-économique de l'apport externe de technologie ont empêché une grande majorité des entreprises du secteur d'Etat de tirer parti de manière optimale des technologies étrangères (3).

\section{UNE INDUSTRIE EN DEVELOPPEMENT RAPIDE}

En 1978, à la veille de la politique d'ouverture, l'industrie électronique chinoise était principalement orientée vers un usage militaire. La production totale en 1980 ne dépassait pas 10 milliards de yuans. Le secteur de l'électronique grand public était sous-developpé en raison du bannissement politique des produits de consommation de masse, jugés « bourgeois » et contre révolutionnaires par les dirigeants de la Révolution culturelle. Les entreprises d'Etat et collectives assuraient $100 \%$ de la production. Celle-ci était essentiellement concentrée à Shanghaï, Pékin et dans la province du Jiangsu, même si d'importants investissements furent réalisés dans les provinces de l'intérieur, Sichuan, Guizhou et Shaanxi dans le cadre du programme du " troisième front » développé durant la Révolution culturelle dans la crainte d'une attaque étrangère sur les bases industrielles du littoral. Malgré la rupture sino-soviétique en juillet 1960 , 
l'industrie électronique chinoise demeure fortement influencée par l'Union soviétique, aussi bien sur le plan technologique que des structures d'organisation de la R\&D.

Quinze années après le début de la politique d'ouverture, quelques statistiques révèlent un développement impressionnant de l'industrie électronique en Chine. La production totale a été multipliée par 12 avec près de 120 milliards de yuans en 1993 (voir fig. 1). La même année, un peu plus de $25 \%$ de la production était exportée, la Chine devenant un des dix plus importants fournisseurs de produits électroniques des Etats-Unis. Ce développement exponentiel s'est articulé essentiellement autour de la croissance de la production dans l'EGP et notamment autour de l'industrie des téléviseurs (tubes cathodiques, composants pour téléviseurs). La Chine dispose désormais de 124 producteurs de téléviseurs, produisant 13 millions de téléviseurs noir et blanc et 13 millions de téléviseurs couleur en 1993. La même année, sept des plus grandes entreprises du secteur électronique étaient des producteurs liés à l'industrie des téléviseurs.

Source : Zhongguo Dianzi Gongye Nianjian

(Annuaire de l'industrie électronique chinoise), 1994, 1990, 1987.

FIG. 1. - Evolution de la production électronique en Chine 1981-1993

L'industrie informatique émerge difficilement même si quelques histoires à succès existent avec l'entreprise Sitong dans les années 1980 et surtout le groupe Legend (Lianxiang, une coentreprise sino-hongkongaise) au début des années 1990, qui se classe au deuxième rang parmi les plus grandes entreprises de l'industrie électronique en Chine. Les produits étrangers dominent très largement le marché, soit par les licences de distribution accordées par des firmes étrangères à des firmes chinoises (c'est le cas du groupe Legend qui réalise une bonne partie de son chiffre d'affaires par le monopole en Chine de la distribution d'ordinateurs personnels étrangers), soit par l'importation : $70 \%$ des ordi- 
nateurs vendus dans la Silicon Valley de Pékin à Zhongguancun sont des produits importés ; $90 \%$ des logiciels, $80 \%$ des imprimantes, $80 \%$ des photocopieurs, $100 \%$ des télécopieurs, $100 \%$ des disques durs et des microprocesseurs sont également importés.

L'industrie des composants est dominée par la production de composants passifs où les producteurs chinois gagnent progressivement des parts de marché sur les marchés internationaux. En 1994, les exportations de composants passifs ont totalisé 2,1 milliards de dollars, soit $50 \%$ d'augmentation par rapport à 1993. En ce qui concerne les circuits intégrés, dont la maîtrise de la production est hautement stratégique car entrant dans la fabrication de la plupart des produits électroniques, la situation apparaît beaucoup plus critique. Les entreprises nationales ont le plus grand mal à suivre une évolution technologique mondiale accélérée. Seule l'entreprise Huajing de Wuxi, où l'Etat a englouti d'énormes sommes d'argent depuis la moitié des années 1980, est actuellement capable de produire des circuits à très haute intégration (VLSI). La première entreprise du secteur est une coentreprise sino-étrangère, la Belling Company de Shanghaï, fondée par Alcatel Shanghaï pour répondre à ses propres besoins de circuits intégrés pour télécommunications. Les entreprises étrangères installées en Chine assurent $53 \%$ de la production nationale qui, elle, ne couvre que $20 \%$ de la demande nationale estimée à près de 1,3 milliard de dollars ${ }^{2}$. Plusieurs tentatives de constitution de grandes entreprises nationales dans l'industrie des circuits intégrés inspirées de l'expérience sud-coréenne se sont soldées par des échecs. C'est dans ce secteur que les transferts de technologie ont été les moins réussis, notamment à Shanghaï.

Le réseau des télécommunications est l'un des plus sous-développés au monde avec 12 millions de lignes en 1992, soit moins de deux téléphones pour 100 habitants, et constitue un frein au développement économique chinois. L'Etat prévoit d'investir 360 milliards de yuans entre 1996 et 2000 pour multiplier par 15 les lignes existantes. Aucun des constructeurs nationaux n'est capable de répondre à ce défi technologique. Le ministère des Postes et Télécommunications dispose d'un monopole d'exploitation, même si récemment des autorisations d'exploitation de réseaux ont été données à son concurrent, le ministère de l'Electronique. En fait, seuls les constructeurs étrangers sont capables de fournir les technologies requises ; ils négocient leur entrée sur ce marché du siècle par le biais de transferts de technologie et d'investissements directs. Les constructeurs européens, avec en tête Alcatel Shanghaï ( $40 \%$ du marché), se taillent la part du lion sur le marché de la commutation publique. Concernant la téléphonie sans fil et les réseaux locaux, malgré une présence importante des leaders mondiaux, Hongkong et Taiwan sont de plus en plus actifs. La situation sur ces marchés devient chaotique, le ministère des Postes et Télécommunications ne contrôlant presque rien des négociations avec les firmes étrangères, les provinces s'octroyant une autonomie de plus en plus importante sans se préoccuper des effets de disparités technologiques à l'échelle nationale.

1. Ne comprend pas les produits fabriqués en Chine par les producteurs étrangers.

2. Zhongguo Dianzi Gongye Nianjian (Annuaire de l'industrie électronique chinoise), 1994, p. III-58. 
La répartition géographique de la production a également évolué. La province du Guangdong est désormais de loin le premier producteur de produits électroniques. Elle passe de la sixième position en 1982 avec 549 millions de yuans (loin derrière Shanghaï et la province du Jiangsu dont la production totale se montait respectivement à 2,2 et 1,6 milliards de yuans), à la première position en 1993 avec 25 milliards de yuans (soit près de trois fois la production actuelle de Shanghaï). Les provinces du Guangdong et du Fujian sont désormais les principales bases exportatrices nationales, exportant respectivement $38,5 \%$ et $32,4 \%$ de leur production. Les autres provinces, à l'exception de celle du Zhejiang qui exporte $23 \%$ de sa production, voient leur part de la production à l'exportation plafonner à moins de $10 \%$.

L'émergence des provinces du Guangdong et du Fujian comme principales bases productives et exportatrices du pays est directement liée à la délocalisation massive des activités intensives en main-d'œuvre de l'industrie électronique de Hongkong (dans le Guangdong) et de Taiwan (dans le Fujian). Il est maintenant établi que la réduction progressive de l'excédent commercial de Hongkong et de Taiwan vis-à-vis des Etats-Unis et la croissance de l'excédent commercial sino-américain proviennent en grande partie de la vague de délocalisation massive dans ces deux territoires en Chine durant les années 1980.

On recense actuellement 432 coentreprises sino-étrangères opérant sur le territoire chinois dans l'industrie électronique. Elles représentent $21 \%$ de la production totale ( $3 \%$ en 1985) et assurent près de $55 \%$ des exportations. Là encore le rôle de Hongkong et de Taiwan est prédominant puisque $60 \%$ des investissements étrangers ont été effectués par ces deux territoires, assurant $9 \%$ de la production totale et $25 \%$ des exportations'. Les statistiques chinoises n'indiquent pas la part des investissements directs japonais dans le total des investissements directs étrangers réalisés dans l'industrie électronique en Chine. Cependant, si l'on se fonde sur la part des investissements japonais réalisés en Chine toutes industries confondues, les investissements asiatiques (Japon, Hongkong et Taiwan, auxquels il faudra ajouter bientôt la Corée du Sud qui développe une politique d'investissement agressive notamment dans la province du Shandong) devancent de très loin ceux des Etats-Unis et de l'Europe avec près des deux tiers des coentreprises installées sur le territoire chinois et assurent $80 \%$ de la production hors secteur d'Etat.

On mesure ainsi le chemin parcouru depuis 1978. Ce développement rapide de l'industrie électronique en Chine n'aurait pas été possible sans un recours massif aux importations de technologies ; mais celles-ci sont encore loin, en ce qui concerne les entreprises d'Etat, d'avoir déclenché un cercle vertueux d'accumulation de capacités technologiques dans le cadre d'une industrie électronique en cours de mondialisation rapide. 
LE BILAN DE LA POLITIQUE D'IMPORTATION

DE TECHNOLOGIE 1978-1991

Les entreprises d'Etat ont essentiellement favorisé un transfert de technologie de type conventionnel : achat de licence, achat d'équipements productifs avec assistance de l'émetteur, achat d'usines clef en main et achat du type « produit clef en main "'. Notre enquête s'est focalisée sur un certain nombre de critères susceptibles d'appréhender la qualité dans l'appropriation des technologies étrangères : retombées commerciales, et plus spécialement les ventes à l'exportation car mieux à même de refléter un réel niveau de performances (prix et qualité) d'utilisation des technologies; qualité des produits; intensité des transformations dans l'organisation du travail et de la gestion de l'entreprise induites par le transfert ; rythme des adaptations / évolutions de la technologie transférée ; continuité et progression du rythme de l'innovation technologique ; diffusion de capacités technologiques à l'extérieur de l'entreprise; répercussions sur la capacité technologique nationale.

\section{Retombées à court terme sur le marché intérieur}

Il est difficile de chiffrer les retombées commerciales de la vague d'importation de technologies et équipements étrangers sur l'ensemble de l'industrie électronique. Il demeure en effet hasardeux de juger de l'efficacité commerciale de certains transferts dans un système économique encore très largement bureaucratisé. Les manipulations administratives des prix, la contrainte de budget douce sont susceptibles de masquer un nombre important de projets inefficaces. Les statistiques chinoises retiennent cependant comme critères le montant des devises épargnées et le montant du chiffre d'affaires supplémentaire attribué au transfert de technologie. Toutes industries confondues, quelques chiffres ont été avancés. Le ministère du Commerce extérieur considère qu'entre 1978 et 1987, 30000 projets d'introduction ont été réalisés pour un coût total de 13 milliards de dollars. Ils auraient rapporté, à la fin des années 1980, 110 milliards de yuans supplémentaires (plus de 25 milliards de dollars US), soit environ $12 \%$ de l'augmentation totale de la valeur de la production sur la même période. Au niveau de l'industrie électronique, plus de 2000 projets d'introduction auraient vu le jour durant les années 1980 pour un coût total de 18 milliards de yuans (dont 3 milliards de dollars us) ${ }^{2}$. Nous n'avons pas eu connaissance des statistiques globales pour l'ensemble de l'industrie sur la contribution et les retombées économiques des projets de trans-

1. Estimations effectuées à partir de Zhongguo Dianzi Nianjian, 1986 et 1990 (Annuaire de l'industrie électronique chinoise).

2. Keji Ribao, 4 février 1991 (Quotidien des sciences et techniques). 
ferts. Les transferts seraient donc à l'origine de la croissance des résultats commerciaux à partir de 1984, jusqu'aux années 1988-1989, en permettant aux entreprises de répondre de manière rapide à la forte demande des marchés de biens de consommation.

Les informations obtenues sur les entreprises d'Etat confirment cependant un phénomène. La plupart des entreprises ayant réalisé un transfert au début des années 1980 ont réussi à tirer parti de la croissance soutenue sur les marchés de l'électronique grand public durant la période 1983-1987, puis on assiste à une dégradation rapide des résultats financiers des entreprises à partir de 1987-1988. Au début des années 1990 dans le secteur électronique, $50 \%$ des entreprises d'Etat étaient jugées inefficaces ; $30 \%$ ayant des performances moyennes ; seules $20 \%$ des entreprises pouvaient être considérées comme performantes au regard d'une batterie de critères allant des résultats financiers à la productivité du travail, en passant par les performances à l'exportation'. Cette évolution confirme d'ailleurs celle de tout le secteur d'Etat, toutes industries confondues.

\section{Croissance limitée des exportations}

Une des principales transformations de l'industrie électronique chinoise repose sur sa participation croissante aux échanges mondiaux. Peut-on conclure cependant que les entreprises chinoises ont réussi à tirer parti des transferts de technologie pour se positionner de manière active sur les marchés internationaux?

La réalité apparaît un peu plus complexe. On assiste plutôt à une dualité du fonctionnement du tissu industriel qui limite considérablement les résultats à l'exportation des entreprises d'Etat. La croissance des exportations chinoises de produits électroniques s'articule principalement autour d'un vecteur : produits à faible contenu en valeur ajoutée, sous-traitance internationale, coentreprises hongkongaises et taiwanaises implantées dans les provinces côtières.

L'insertion des entreprises d'Etat dans le commerce international est beaucoup plus problématique. Les entreprises d'Etat de notre échantillon, exceptée une d'entre elles qui avait développé des liens de sous-traitance avec une entreprise japonaise dans le domaine des tubes cathodiques noir et blanc, considéraient le marché intérieur comme prioritaire. Celles opérant dans l'électronique grand public exportaient entre 5 et $10 \%$ de leur production; pour celles situées dans les autres sous-secteurs, les exportations étaient quasiment inexistantes.

1. Zhongguo Renmin Daxue Gongye Jingjixi, Jijie Dianzi Gongyebu Shengchangsi, Jidian hangye guoyou daxing qiye huoli wenquan diaocha fenxi (Analyse du questionnaire d'enquête sur la vitalité des grandes entreprises d'Etat du secteur électronique et des machines), in Guanli Shijie, n' 2, 1993, p. $148-152$. 


\section{Poursuite et créations de distorsions dans la structure industrielle}

Un des traits les plus saillants de la politique de transferts de technologies dans les années 1980 reste certainement le fait que les achats de technologies étrangères se sont déroulés de manière disséminée et répétée. Ce phénomène se reproduit pour tous les produits qui composent la branche électronique. Les achats les plus nombreux touchent tout particulièrement les activités en aval des filières de production, là où les entreprises sont en contact direct avec les réseaux de distribution'. La nature de ces importations n'est pas sans occasionner déséquilibres et déficiences sur le plan du développement sectoriel :

- La reconduction à l'identique de l'effectif pléthorique des entreprises du secteur jusqu'en 1989 a été en partie alimentée par l'achat de technologies étrangères. Chaque province a de manière séparée, sans se soucier des effets sur le développement national, favorisé la modernisation de l'outil de production des entreprises dont la gestion lui incombait. Alors que la taille du marché intérieur s'y prêtait tout particulièrement, les impératifs d'économie d'échelle et de " gamme ", qui demeurent pourtant cruciaux dans l'industrie électronique des années 90, ont été totalement ignorés (Chang Weimin, 1991). Le transfert peut donc également être analysé comme un moyen de survie et de pérennité d'un tissu d'entreprises inefficaces, la Chine ayant sur ce point hérité d'une structure industrielle caractérisée par la non-spécialisation des grandes, mais également des petites entreprises (Daerquan xiaoerquan).

- Le développement incontrôlé de ce type d'investissement a provoqué à partir de 1988 d'importantes capacités productives excédentaires. L'augmentation considérable des stocks et méventes ainsi que la détérioration des comptes financiers des entreprises d'Etat dont nous avons mentionné l'existence ci-dessus sont directement liées à ce phénomène.

- La forte proportion de projets portant sur l'établissement d'une production en aval des filières, notamment dans l'EGP, a créé de graves goulots d'étranglement en amont. La balance commerciale électronique indique un poids de plus en plus important des importations des pièces détachées et matières premières qui servent à alimenter les productions en aval issues des transferts (tubes cathodiques pour téléviseurs, circuits intégrés et autres semiconducteurs).

1. Le cas le plus connu et qui a décidé le gouvernement central chinois à contrôler les importations de technologies étrangères, est celui des téléviseurs : entre 1978 et 1988 pas moins de 120 chaînes de montage de téléviseurs dont $90 \%$ sont d'origine japonaise ont été achetées à l'étranger par près d'une centaine d'entreprises. Dans le domaine des postes radios et magnétophones, on recense entre 1978 et 1985 , plus de 120 projets d'introduction de technologies et d'équipements étrangers. C'est également le cas pour l'électroménager : 85 chaînes de production de réfrigérateurs entre 1979 et 1985, 70 chaines de montage et de fabrication de cassettes audio et vidéo et 160 chaines de fabrication de semi-conducteurs dont 45 chaines de fabrication circuits intégrés. 
Transformations limitées dans les capacités technologiques des entreprises

La presse spécialisée monte régulièrement en épingle les réussites d'entreprises dans la voie qui les conduit de l'importation de technologies étrangères à l'innovation endogène, en passant par un processus d'assimilation progressive. On notera que dans la plupart des cas, seule la performance technique est prise en compte alors que les critères d'efficacité des transferts sont fondés de plus en plus, même pour des pays comme la Chine et en raison d'une globalisation croissante de l'industrie électronique, sur une rentabilité économique se définissant par rapport au marché mondial.

Si l'on considère l'échelle des capacités technologiques d'une entreprise présentée dans les travaux de S. Lall (1990) ou de K. Dalhman et al. (1987), ainsi que la complexité croissante dans l'accumulation de capacités technologiques dans les succès des NPI asiatiques (P. Judet, 1989 ; K. Dalhman et al., 1987), nos études de cas font apparaître l'impuissance des entreprises d'Etat à entamer un cercle vertueux d'accumulation de capacités technologiques. La majorité des entreprises a rencontré d'importantes difficultés dès les stades les plus élémentaires, à savoir dans les capacités de production nécessaires à une utilisation efficace des technologies. En premier lieu, les entreprises ont failli à l'entretien et la réparation des équipements; les contrats de maintenance avec des firmes étrangères ont été difficiles à supporter financièrement pour les entreprises d'Etat disposant de peu de devises étrangères ; un grand nombre se livrait alors à du bricolage-maison limitant les capacités techniques des équipements.

Plus gravement, $90 \%$ des transferts de technologie ont porté sur des contrats de type " produits clefs en main » dans des activités d'assemblage avec une assistance technologique étrangère limitée (phases de montage et de lancement de la production). La focalisation sur des critères productifs a en effet contribué à vider une bonne partie du contenu soft des contrats de transfert, limitant les opportunités d'apprentissages technologiques sur le plan organisationnel, occasionnant des effets en retour négatifs quant à l'efficacité de l'appropriation des technologies étrangères'. Ces caractéristiques ne créent pas en effet les conditions optimales d'appropriation de systèmes technologiques allogènes ; elles entrent en contradiction avec les fortes interdépendances existant entre les différents éléments constitutifs des capacités technologiques (équipement, savoir faire, organisation), et qui les rendent bien souvent insécables sans une perte d'efficacité pour le récepteur de technologie.

Enfin, sur le plan de la diffusion dans le tissu industriel local de nouvelles capacités technologiques, l'adaptation des fournisseurs locaux aux exigences des technologies étrangères s'est en général révélée lente et difficile. D'impor-

1. Ce phénomène a été ressenti gravement dans les transferts de technologie concernant l'industrie des circuits intégrés. Une enquête chinoise de la Commission d'Etat au Plan révélait, en 1988, que sur près de 45 chaînes de production importées, seules 4 fonctionnaient au niveau de capacité attendu lors de l'établissement des contrats de transfert. Dans les usines de Shanghaï, les taux de rebuts atteignaient, en 1988, $50 \%$ en moyenne pour les circuits à faible intégration et presque $90 \%$ pour ceux à moyenne et haute intégration. 
tantes fluctuations en terme de qualité, de prix, de livraisons de la part des fournisseurs locaux ont handicapé les entreprises dans le respect des normes de qualité des produits.

\title{
Importation de technologie \\ "versus " capacité nationale de la R\&D
}

Au niveau méso-industriel, on observe un problème de couplage entre les projets d'introduction de technologies étrangères et le système de R\&D national : une étude réalisée en 1989 par le Bureau des introductions de technologies étrangères de la Commission d'Etat des sciences et techniques révélait que $63 \%$ des résultats des instituts de recherche étaient dédoublés par des importations de technologies étrangères'. Ce phénomène contribue à diminuer la rentabilité de l'effort de R\&D national dans un pays où les ressources financières sont rares, mais également à renforcer la dépendance technologique dans les activités de conception où les firmes chinoises disposent de faibles ressources internes (in house). Il y a donc pérennisation du cloisonnement entre le système productif et celui de la R\&D. De ce fait, une grande partie des nouveaux produits introduits sur le marché sont issus d'un achat de technologies étrangères. En 1988, dans la province du Guandong, $80 \%$ des 500 produits nouvellement introduits sur le marché reposaient sur des capacités de production et de technologies achetées à l'étranger'. On assiste ainsi à une corrélation étroite, d'une part entre la nature des achats de technologies étrangères (visant à établir des capacités de production) et les faibles ventes des résultats des instituts de recherche (souvent inadaptés pour un transfert dans la sphère productive), et d'autre part entre les difficultés dans le processus d'assimilation des technologies étrangères par les entreprises chinoises et la faible participation des instituts de recherche nationaux au processus d'introduction (instituts qui concentrent cependant une bonne partie de la capacité technologique humaine du pays). La priorité accordée dans le VIII ${ }^{e}$ Plan (1991-1995) au travail d'assimilation des technologies étrangères fournit la preuve d'une reconnaissance, de la part des autorités, des piètres résultats en ce domaine ainsi que de l'urgence du problème pour le développement industriel futur.

\author{
LES OBSTACLES DANS L'ACCUMULATION \\ DE CAPACITÉS TECHNOLOGIQUES
}

\section{Poursuite d'une régulation bureaucratique des entreprises d'Etat}

Le transfert de technologie est un contrat qui lie deux entreprises sur la vente d'une combinaison de matériels et d'informations se rapportant à une partie, voire à l'ensemble de l'activité d'une entreprise (de la R\&D à la commer-

1. Yinjin Yu Zixun, mai 1989 (Introduction (de technologies étrangères) et conseil)

2. Guoji Dianzibao, 23 mai 1988 (Journal de lélectronique internationale). 
cialisation), et permettant à une firme de commercialiser un produit dans certaines conditions de coûts. Cependant ce contrat ne débouche pas automatiquement sur l'acquisition de nouvelles capacités technologiques pour le récepteur. Cette confusion entre transfert de technologie et acquisition de nouvelles capacités technologiques est certainement à la source de bon nombre d'échecs qui ponctuent l'histoire des transferts dans les PVD. L'analyse des revers et des réussites - notamment dans les NPI asiatiques - mais également l'intérêt croissant des économistes concernant le développement des capacités technologiques des entreprises et des nations ont permis de mieux comprendre la complexité du processus d'acquisition de nouvelles capacités par le transfert de technologie. Celle-ci ne résulte pas uniquement du machiavélisme des pays industrialisés et de leur volonté de perpétuer une dépendance économique pour les PVD ; par définition, le transfert de technologie est un processus de diffusion entravée' : il s'agit en effet pour le récepteur de s'approprier les capacités technologiques existantes dans le système technologique allogène. Cette appropriation est subordonnée au respect de certaines règles d'apprentissage combinées à un effort endogène permettant de multiplier les occasions d'apprentissage technologique ${ }^{2}$. Assurément ces conditions n'ont pas été réunies dans le cas des entreprises d'Etat chinoises.

Bon nombre de blocages dans l'innovation et l'appropriation des technologies étrangères par les entreprises chinoises d'Etat dans les années 1980 relèvent du fonctionnement spécifique du secteur d'Etat. La mise en place des réformes dans la gestion des entreprises d'Etat après 1978 a certes permis de relâcher un certain nombre de contraintes systémiques dans le fonctionnement des entreprises et d'améliorer les conditions dans lesquelles elles réalisent l'appropriation des technologies étrangères. Cependant les réformes ont abouti à des résultats inégaux en matière d'autonomie décisionnelle des entreprises. Si l'on observe l'émergence d'une véritable autonomie en matière de fixation des prix et de commercialisation, l'investissement, le financement, la gestion des ressources humaines et la liberté de commercer à l'étranger restent sous l'emprise d'un important contrôle bureaucratique. Les réformes ont surtout contribué à faire évoluer les rapports de pouvoir sur les entreprises au sein de la bureaucratie, en transférant une bonne partie des prérogatives de gestion des entreprises du pouvoir central aux pouvoirs provinciaux, voire municipaux ; les entreprises disposent toujours, quant à elles, d'une autonomie décisionnelle très réduite concernant bon nombre de décisions stratégiques, prolongeant ainsi les blocages structurels issus de la période antérieure aux réformes.

1. Composantes tacites individuelles et collectives qui dépendent également d'éléments externes à l'entreprise comme les rapports de sous-traitance ou lorganisation du systeme national de R\&D, des capacités technologiques des firmes difficilement transférables, rôle stratégique des technologies dans la compétition industrielle impliquant une gestion conséquente de la part des entreprises émettrices.

2. Progressivité dans le degré de complexité des connaissances technologiques acquises, continuité dans l'effort technologique, choix des technologies et des modalites de transfert. 


\section{a / La négation des ressources humaines}

Le système du «Bol de riz en fer » (Tiewanfan) en vigueur dans les entreprises d'Etat durant la période maö̈ste, qui garantissait l'emploi à vie, reste encore en vigueur. Le système de transmission de l'emploi entre membres d'une même famille ( $D i n g t i$ ) demeure encore largement répandu. Plusieurs réformes, comme la création de " centres d'échanges de talents " pour le personnel qualifié ou la loi de 1986 sur l'établissement de contrats à durée déterminée pour tous les nouveaux employés du secteur d'Etat, ont tenté progressivement de briser le système du «Bol de riz en fer ». Les résultats sont encore très limités. A la fin des années 1980, seul 1,3\% des quelque 100 millions d'employés du secteur d'Etat ont changé de travail au cours d'une année. Les transferts entre entreprises d'Etat restent de lourdes procédures administratives tant pour celui qui désire changer de travail que pour le nouvel employeur qui recherche du personnel qualifié (M. Korzec, 1992). Les difficultés d'obtention de personnel qualifié renforcent les tendances à la " thésaurisation » des ingénieurs et gestionnaires, les entreprises préférant sous-utiliser leurs personnels qualifiés plutôt que de les laisser partir. Les rigidités du système en matière de gestion des ressources humaines posent ainsi de graves problèmes pour les entreprises, aussi bien sur le plan de l'efficacité financière en raison des sureffectifs, mais également pour la mise en place d'une véritable stratégie de formation, de recrutement et de mobilisation du personnel nécessaire aux apprentissages technologiques lors des transferts et, de manière plus générale, à toutes les activités se rapportant à l'innovation.

\section{b / La faiblesse des ressources propres destinées à l'innovation}

Parallèlement, d'importants blocages proviennent de la faiblesse des ressources financières des entreprises d'Etat destinées au développement de capacités technologiques. Une des principales réformes des années 1980 réside dans la possibilité pour les entreprises d'Etat de conserver une partie des bénéfices de leur activité (Liucheng). Sur le plan du transfert de technologie, ceci devait pouvoir permettre aux entreprises de disposer de plus de souplesse dans l'affectation de ressources pour l'achat et l'assimilation des technologies. La réalité apparaît fort différente ; dans l'industrie électronique à la fin des années 1980 , les entreprises d'Etat disposaient en moyenne, pour leur autofinancement, de 6 yuans sur 100 yuans de bénéfices réalisés. Les résultats officiels communiqués par le ministère de l'Electronique début $1991^{1}$, indiquent que les bénéfices gardés par les entreprises du secteur se montaient en 1990 à près de 680 millions de yuans, soit à peine $1 \%$ du total du chiffre d'affaires pour le secteur. Différentes analyses s'accordent ainsi à reconnaître le manque de ressources des entreprises chinoises sur le plan du supplément de capitaux circulants et de capitaux fixes

1. Zhongguo Jidianbao, 12 mars 1991 (Journal de l'industrie chinoise des machines et de l'électronique). 
occasionnés par l'introduction de la technologie étrangère. Pour la ville de Shanghaï, R. Conroy avance un montant consacré à l'assimilation des technologies étrangères égal à $1 / 40^{e}$ du total dépensé lors de l'achat. Les experts chinois se plaisent à comparer la Chine et le Japon quant aux proportions financières consacrées au travail de digestion/assimilation par rapport au montant initial de l'achat : pour $1 \mathrm{~F}$ d'achat de technologie étrangère, le Japon dépense en moyenne $10 \mathrm{~F}$ pour le travail d'assimilation, alors que dans le cas chinois, cette somme était en 1988 de l'ordre de 7 centimes' ! Derrière ce chiffre se profile une différence fondamentale avec l'histoire du développement technologique au Japon : les entreprises japonaises depuis l'époque Meiji ont consacré non seulement beaucoup de ressources financières pour l'adaptation des technologies transférées, mais elles ont également cherché à adapter leur organisation (circulation intense et horizontale des informations) afin de répondre aux impératifs de modification des produits et des procédés de production inhérents à tout transfert de technologie. Ce faisant elles ont développé des capacités d'innovation mineure en réalisant une ouverture du "paquet technologique ", mais aussi en considérant l'organisation comme une dimension inséparable des apprentissages technologiques (M. Fruin, 1994).

La faiblesse des ressources destinées au progrès technologique dans les entreprises d'Etat provient essentiellement de trois phénomènes qui reflètent les limites des réformes dans le secteur d'Etat. On constate tout d'abord une dégradation dans le bénéfice des entreprises d'Etat tous secteurs industriels confondus. La rentabilité financière des entreprises s'est dégradée structurellement au cours des années 1980 avec une accélération à la fin des années 1980. Les explications sont assurément multiples; cependant, les importations de lignes de production étrangères, leur dissémination sur un nombre important d'entreprises empêchant l'apparition d'économies d'échelle, la faible qualité des produits ont favorisé l'émergence d'une forte capacité de production excédentaire ne répondant pas toujours aux exigences du marché ${ }^{2}$. Bon nombre d'entreprises d'Etat sont de cette manière plongées dans un cercle vicieux : actrices et victimes des importations non coordonnées de technologies étrangères, elles ne sont pas en mesure de dégager des bénéfices suffisants à la fois pour l'assimilation des technologies, mais surtout pour engranger un rythme régulier de transformations technologiques qui, si l'on se réfère aux expériences des NPI asiatiques, conditionne en partie l'accumulation de nouvelles capacités technologiques (K. Dalmhan et al., 1987).

Ensuite, on assiste à une utilisation discrétionnaire de la fiscalité par la bureaucratie. L'impôt a plutôt été utilisé comme un outil d'égalisation des différences de profitabilité intra et intersectorielle (Kang Chen, 1990), un instrument de régulation de situations déficitaires, voire, au pire, comme un instrument

1. Keji Ribao, 12 juin 1991 (Quotidien des sciences et techniques).

2. En 1989, près de $45 \%$ du potentiel de production de téléviseurs resterait inutilisé. De récentes études chinoises ont estimé que plus de la moitié des chaînes de fabrication de téléviseurs fonctionnaient en dessous de la moitié de leur capacité (D. F. Simon, 1991). 
d'enrichissement de la bureaucratie locale. L'utilisation concrète de la politique fiscale a contribué au relâchement de la contrainte de budget de l'entreprise ; celle-ci, par l'intermédiaire de marchandages auxquels donne lieu la fixation du niveau des taxes, peut, en accord avec la bureaucratie, manipuler le niveau de sa profitabilité ex-ante, provoquant ainsi la poursuite de comportements d'irresponsabilisation face aux investissements réalisés propres aux entreprises dans les économies socialistes où la faillite n'existe pas.

Enfin, il apparaît que les ressources propres des entreprises n'ont pas été orientées sur des activités de modernisation technologique. L'utilisation officielle des bénéfices de l'entreprise subit en effet d'importantes modifications au contact de la réalité du fonctionnement de l'entreprise d'Etat. La règle posée par les réformes en matière d'utilisation des bénéfices par les entreprises est celle des « $5,3,2 »: 50 \%$ pour le fond de modernisation (qui doit alimenter l'innovation des entreprises), $30 \%$ pour le fond social, $20 \%$ pour les bonus salariaux. En pratique les entreprises ont adapté cette règle. L'entreprise d'Etat chinoise a été en effet pendant longtemps le vecteur privilégié du développement du système de l'unité de travail (Danwei). Ce mode d'organisation sociale de l'économie urbaine chinoise, courroie de transmission du pouvoir politique communiste, est incontournable dans toute recherche portant sur le fonctionnement et la réforme des entreprises d'Etat chinoises. En récupérant l'ensemble des fonctions sociales (logement, retraite, assurance maladie) une bonne partie des ressources de l'entreprise est détournée de la fonction productive. Tout indique que les choix des directeurs d'unité de travail se sont réalisés aux dépens de la modernisation technologique (décision par essence risquée) et plutôt en faveur d'une amélioration du cadre de vie (salaires inclus) du collectif de l'unité de travail. Les fonctions extra-économiques de l'unité de travail chinoise ont primé : logement, couverture sociale, bonus pour le collectif ouvrier déconnecté des performances réelles des entreprises. Le niveau très faible de l'amortissement n'a pas compensé ce phénomène. Le taux d'amortissement moyen des équipements industriels dans le secteur d'Etat en 1988 est d'environ 5,3\%'. La préférence pour l'investissement de capacité au détriment de l'innovation demeure, même après les réformes, une des caractéristiques principales du fonctionnement de l'investissement des entreprises d'Etat. La diffusion du contrat forfaitaire à l'ensemble des entreprises d'Etat ne semble pas avoir modifié l'hypothèse de J. Winiecki (1988) concernant l'aversion au risque de la part des chefs d'entreprise pour l'innovation dans un système socialiste. Ceux-ci demeurent particulièrement rétifs à toute modification substantielle des paramètres de production non couverts par le Plan; or même si les réformes ont théoriquement presque supprimé l'existence des plans impératifs, les entreprises restent extrêmement dépendantes des différents bureaux industriels pour tout ce qui concerne leur approvisionnement en matières premières et composants. De

1. Qui plus est, ces fonds ne sont pas intégralement conservés par les entreprises. La loi de 1985, puis celle de 1986, qui réglementent l'utilisation des fonds d'amortissement laissent une marge de manœuvre à l'administration. On estimait en 1990 que pour 100 yuans d'amortissement, une fois réglées les diverses taxes, l'entreprise peut réellement disposer de 50 yuans pour le fond de modernisation. 
plus, dans le contexte du système des contrats forfaitaires, les dirigeants sont conduits à maximiser les profits à court terme et à répondre aux exigences de croissance du pouvoir d'achat du collectif ouvrier, ceci aux dépends de choix conséquents d'investissements permettant aux entreprises de déployer une stratégie de développement à long terme, notamment technologique. L'insuffisance chronique en ressources financières dont souffrent les entreprises d'Etat concernant le fond de modernisation les poussent ainsi à se tourner vers la recherche de financement extérieur.

\section{c/ Les effets pervers du système d'investissement}

De manière générale, les entreprises d'Etat souffrent d'une double dépendance à l'égard de la bureaucratie en matière d'investissement. D'une part, on assiste à une dépendance légale en matière décisionnelle. Malgré une réduction des rigidités, pour tout investissement, les entreprises doivent passer au travers d'une longue série d'approbations de la part des autorités de tutelle' concernant l'étude de faisabilité, le financement, les garanties dans l'approvisionnement des inputs nécessaires à la bonne réalisation du transfert, etc. Or même si les refus de l'administration sont rares, le processus décisionnel concernant le transfert s'en trouve considérablement allongé et compliqué, limitant d'autant la saisie d'occasions commerciales sur le marché.

D'autre part, on note une dépendance financière vis-à-vis de la bureaucratie qui débouche sur des blocages encore plus lourds de conséquences. Les réformes ont certes considérablement réduit l'importance des dotations budgétaires gratuites, mais l'augmentation de l'autofinancement et du recours aux crédits bancaires n'a pas atténué la dépendance des entreprises par rapport à la bureaucratie dans leurs décisions d'investissement. Nous avons pu observer ci-dessus la faiblesse de l'autofinancement concernant les activités de modernisation de l'entreprise. Le recours aux crédits bancaires a considérablement augmenté, représentant presque $30 \%$ des financements des entreprises d'Etat dans leurs investissements de modernisation. Néanmoins, cette transformation est captieuse, si l'on se réfère à une autonomie décisionnelle croissante de l'entreprise. Le système bancaire est en effet entièrement contrôlé par l'administration. Les crédits bancaires ne sont pas attribués en fonction d'une évaluation de la viabilité financière du projet. Ils demeurent un instrument de financement quasi identique à la dotation budgétaire, étant donné la contrainte de remboursement très "douce " qui pèse sur les entreprises (Chang Dakai, 1989). Les décisions d'octroi de crédits demeurent à la discrétion de la bureaucratie locale, en raison des " relations semi-coercitives " de la part des planificateurs et des responsables politiques locaux mais également, de manière plus globale, en raison d'une étroite collaboration entre

1. Les projets d'investissement supérieurs à 30 millions de yuans doivent remonter jusqu'au gouvernement central et être soumis à l'approbation de la puissante Commission d'Etat au Plan. Pour un montant inférieur, les échelons locaux de l'administration sont compétents en la matière. 
bureaucraties locales et banques locales dont l'ultime intention est d'accélérer le développement économique de la localité.

Le financement des investissements concernant les transferts de technologie ne repose jamais uniquement sur un seul type de financement. Les entreprises utilisent une tactique connue dans les autres pays socialistes, notamment en Hongrie, à savoir le recours à un montage entre financement bancaire, dotations budgétaires gratuites, et crédit auprès du partenaire étranger. Dans tous les cas cependant, l'entreprise reste dépendante de la bureaucratie pour l'obtention du financement ; elle est ainsi réduite à développer une stratégie complexe face aux organismes de tutelle, qui la conduit à une série de marchandages avec les différents échelons de la bureaucratie (Huchet, 1993). Au total, même si les dotations budgétaires ont vu leur importance considérablement réduite dans le financement des investissements des entreprises d'Etat, la pérennisation des rapports de dépendance vis-à-vis de la bureaucratie conduit à des effets pervers, similaires à ceux existant dans l'ancien système planifié.

Ces effets constituent des blocages importants pour une appropriation efficace des technologies importées. Une des premières conséquences réside dans le phénomène de saupoudrage des investissements et de sous-évaluation chronique des besoins financiers liés aux projets. Les quotas financiers dont dispose la bureaucratie ne sont jamais suffisants pour répondre aux demandes des entreprises qui ont tendance à demander toujours davantage. La véritable évaluation financière se faisant souvent ex post, ce système entraîne un échelonnement du financement de l'investissement sur plusieurs plans annuels, ou l'impossibilité de mener le projet dans des conditions d'exploitation optimale, faute de moyens financiers. Ceci constitue un handicap de taille dans toute stratégie d'ingénierie à rebours et de transformation/création à partir de la technologie transférée, stratégies qui demandent un investissement supplémentaire très important. L'entreprise exprime ainsi une très forte sensibilité au prix du contrat. Devant l'étroitesse des moyens qui lui sont alloués par la bureaucratie, elle privilégie rationnellement le prix au détriment bien souvent de la part du soft. Corroborant les résultats de notre échantillon, une étude indiquait qu'en 1987 seules $15 \%$ des dépenses de transfert de technologie étaient consacrées au software (Zhang Guoying, 1990). Cette caractéristique freine les possibilités d'une assimilation optimale de la technologie étrangère, notamment sur les aspects organisationnels dont nous avons observé les limites.

Enfin la dernière conséquence réside dans la persistance d'une contrainte de budget douce pour les entreprises d'Etat. Or fondamentalement, même après la suppression progressive des dotations budgétaires, il s'agit toujours, aux yeux des entreprises, de l'argent de l'Etat. La contrainte de budget "douce », s'exprimant sous la forme d'un financement sans obligation ultime de remboursement, demeure un déterminant de l'inefficacité de l'investissement et a fortiori du transfert de technologie. Ce mode de financement freine également toute rationalisation du tissu industriel puisque aucune entreprise n'est véritablement responsable en cas d'un mauvais rendement de l'investissement. Conjugué avec 
l'absence de marché concurrentiel et un protectionnisme vis-à-vis de la concurrence étrangère, le problème de l'efficacité ne constitue pas une urgence pour les entreprises d'Etat.

\section{Déficiences dans la gestion institutionnelle du transfert}

L'Etat peut également favoriser l'appropriation des technologies étrangères par la création d'un environnement favorable aux entreprises permettant le pilotage efficace de l'apport externe de technologies. Le travail d'appropriation mené par les firmes réceptrices engage toute la capacité technologique nationale ; dans cet effort les entreprises ont besoin d'un ensemble de relais externes qui leur permettent aussi bien d'avoir accès à une information sur l'état de l'art mondial des technologies, que de compter sur une amélioration de leur environnement extérieur (système éducatif, marché du travail, accès aux ressources financières, développement et accès aux programmes de R\&D publics, circulation de l'information technologique, etc.). Dans cette délicate mission, l'Etat chinois semble loin d'avoir créé les conditions idéales.

\section{a) Les cloisonnements ministériels}

Il existe une multitude d'acteurs dans l'administration chinoise qui interviennent dans le processus de transfert de technologie. Ces interventions se font sur la base de compétences exclusives en matière de tutelle des entreprises d'Etat et débouchent sur un fait paradoxal : l'absence de perspective nationale en matière de gestion de l'apport extérieur de technologies, et d'une manière plus générale sur l'inhibition de toute volonté stratégique de développement sectoriel à l'échelle nationale. Non seulement l'entreprise est dépossédée d'un pouvoir décisionnel sur la conduite du transfert, mais on assiste également à une sur-représentation quantitative des organismes bureaucratiques intervenant dans le processus de transfert qui agissent sans aucune coordination à l'échelle nationale'. On assiste ainsi à une séparation des responsabilités techniques et commerciales. Le ministère de Tutelle détient l'argent mais ne s'occupe directement ni des négociations avec l'entreprise étrangère, ni du développement technologique. Les négociations sont laissées au ministère du Commerce extérieur par l'intermédiaire des différentes compagnies d'import-export qui sont sous sa responsabilité. Celles-ci donnent la priorité à l'aspect commercial de l'achat, et ne s'occupent qu'accessoirement des répercussions technologiques. Cette séparation existe également en matière d'exportation pour les entreprises d'Etat chinoises et contribue à accentuer la déconnexion déjà importante des entreprises d'Etat avec les marchés internationaux. Les rapports indirects qu'entretiennent

1. D. F. Simon (1989) précise qu'il est difficile de connaitre le total des importations de technologies depuis 1978 : il existe plus de 100 départements au niveau du seul ministère du Commerce extérieur qui détiennent une compétence d'approbation dans l'introduction de technologie. 
ces dernières avec les clients étrangers via les compagnies d'import-export sont de nature à entraver la diffusion d'informations technologiques au bénéfice des entreprises chinoises.

Parallèlement, on assiste à des cloisonnements importants entre les différents "systèmes ministériels » qui interviennent dans l'évolution de l'industrie électronique nationale. Chaque ministère développe, contrôle des unités de production et de recherche en fonction de sa finalité productive; le ministère des Télécommunications, celui de l'Aéronautique ont chacun besoin de produire des semi-conducteurs : ils vont, tout naturellement et sans se préoccuper du gaspillage de ressources et des coûts d'opportunité en matière d'économies d'échelle, dupliquer production et R\&D, et importation de technologies étrangères. Ces cloisonnements sont aussi préjudiciables car les achats de technologies étrangères occasionnent des modifications parfois importantes (maind'œuvre, capitaux circulants, matières premières) ; or ces intrants sont gérés le plus souvent de manière séparée par différents bureaux industriels; dans ce cadre, les entreprises sont dans la nécessité de conserver, voire de rechercher la protection de la bureaucratie dans toute décision technologique modifiant leurs paramètres de production. Ceci contribue de ce fait à une mauvaise planification des projets : pénurie de certains intrants, phénomène de substitution d'intrants qui dégradent la qualité des produits finis, augmentation des coûts, recherche onéreuse de devises pour assurer les importations (à travers des exportations à perte), etc.

Enfin s'ajoutent à cela des difficultés de protection du marché intérieur et de conduite de politiques nationales de développement sectoriel. Certaines entreprises ayant techniquement réussi leur transfert ont vu leur marché intérieur fondre devant les importations de matériels étrangers par leurs clients. L'usine informatique de Huanan à Canton, qui figurait dans notre échantillon, a réussi techniquement le transfert de technologie française du mini-ordinateur "Solar » mais a dû arrêter la production quelques années plus tard, ses clients potentiels importants des mini-ordinateurs DEC, IBM, NEC et Fujitsu, mais également, comble du phénomène, des ordinateurs "Solar " fabriqués en France.

\section{b / Les cloisonnements provinciaux}

On assiste depuis le début des réformes à la poursuite d'un problème connu sous le terme de "cellularisation » de l'industrie chinoise, problème analysé depuis longtemps par A. Donnithorn qui, en 1967, insistait déjà sur la politique d'autosuffisance industrielle menée par les différentes bureaucraties provinciales. En matière de fiscalité, les revenus de l'Etat sont en grande partie assurés par l'impôt sur l'activité économique des entreprises ; cependant le système fiscal est organisé autour d'un partage des recettes entre le gouvernement central et les bureaucraties provinciales. Les provinces sont compétentes pour la levée des impôts et négocient de manière séparée avec le centre un quota de redistribution des recettes fiscales. Les objectifs n'étant fixés que sur un plan financier, 
les provinces ont ainsi, de manière très rationnelle, essayé de maximiser le revenu budgétaire local et d'améliorer le bien-être des résidents locaux (Kang Chen, 1990). Les entreprises chinoises qui ont réussi très rapidement à se positionner sur des marchés - les appareils ménagers, l'EGP, l'alimentaire, les cigarettes - ont pu bénéficier de profits relativement importants. Par l'intermédiaire d'un contrôle sur le système bancaire local, les gouvernements provinciaux ont favorisé une allocation des ressources vers un tel type d'investissement dans l'intention de maximiser les profits fiscalisés. L'enracinement provincial des transferts a ainsi contribué à intensifier la compartimentation géographique du développement industriel. On comprend mieux de ce fait pourquoi le transfert de technologie a été très largement parcellisé, portant sur de faibles capacités de production, mais également "répété 》 (Chongfu) sur l'ensemble des provinces sans une véritable perspective de développement sectoriel à l'échelon national. Les provinces ont favorisé une affectation des ressources sur des capacités productives de montage en aval des filières de production. C'est dans ce cadre qu'il faut analyser le phénomène observé concernant l'importation, entre 1978 et 1985 , de plus de 110 chaînes de montage de téléviseurs. Chaque province possède désormais un, voire plusieurs fabricants de téléviseurs sur son territoire. De manière générale, dans tous les sous-secteurs qui composent l'industrie électronique, on retrouve la même volonté provinciale de maximiser les rentrées fiscales et d'assurer le développement de l'industrie locale, au détriment cependant d'un développement national efficace.

\section{c / Les mirages et les réalités de la politique technologique nationale}

La politique technologique de l'Etat chinois a longtemps été calquée sur le modèle soviétique sans cependant disposer de l'importance des ressources financières et humaines existantes dans l'ancienne URSS. De ce fait, les entreprises d'Etat chinoises ont souffert des caractéristiques structurelles de l'organisation d'un système de R\&D de type soviétique. Un certain nombre d'études, parmi lesquelles celles de P. Hanson et K. Pavitt (1987), et surtout celle de J. Winiecki (1988), ont montré non seulement l'inefficacité structurelle d'un système de R\&D centralisé et planifié, mais également le caractère indissociable qu'il entretient avec le projet politique des pays socialistes. D'une manière générale, les systèmes de R\&D centralement planifiés se sont révélés défaillants en raison de plusieurs caractéristiques. En premier lieu les stimulants : les instituts de recherche et les unités de production fonctionnent sous une contrainte de budget douce ; l'invention est rétribuée par un ensemble de récompenses non financières et reste liée à une notion de prestige plutôt qu'à une utilisation à des fins commerciales. En deuxième lieu une centralisation excessive : les entreprises se révèlent incapables de saisir toutes les occasions technologiques et commerciales en raison d'un processus de décision centralisé et bureaucratisé, d'une absence de pluralisme et d'une planification a priori des activités de recherche appliquée. Enfin la localisation des activités de R\&D à l'extérieur des entreprises 
et une participation très limitée des entreprises au marché international, qui provoquent une déconnexion des activités de R\&D des besoins du marché.

Le système de R\&D chinois n'échappe pas à ce constat. Dès la fin de la Révolution culturelle, conscient de ce problème, le gouvernement central a mis en place une série de réformes : responsabilisation financière des instituts de recherche avec suppression progressive des subventions de l'Etat, autorisation pour les instituts de faire commerce de leurs compétences technologiques et de conserver une partie des bénéfices correspondants, création de «zones de développement pour la haute technologie » (Gaoji jishu kaifa qu), mise en place d'un droit de la propriété intellectuelle, réhabilitation du rôle des intellectuels dans la société, et enfin création d'organes supra-ministériels susceptibles de coordonner l'ensemble de la politique de développement technologique nationale.

Il est évidemment encore trop tôt pour juger définitivement de l'impact des réformes sur le comportement technologique des entreprises d'Etat, cependant les multiples bilans, effectués tant par les autorités chinoises que par les observateurs étrangers, signalent la persistance de dysfonctionnements propres aux systèmes de R\&D de type soviétique, mais également l'apparition de distorsions nouvelles issues du processus réformateur (R. Conroy, 1991; M. Goldman et D. F. Simon, 1989). Derrière la faible efficacité du système de R\&D national, plusieurs phénomènes sont en étroite relation.

On assiste tout d'abord à la persistance d'un environnement structurel non stimulant lié au fonctionnement des entreprises dans le secteur d'Etat dont nous avons évoqué les principaux problèmes ci-dessus. En sus, il faut signaler l'absence de véritable concurrence entre les firmes'. La protection du marché national des importations étrangères, les restrictions imposées aux coentreprises concernant la vente sur le marché chinois, et surtout la protection des marchés provinciaux par les bureaucraties locales freinent l'émergence d'un environnement concurrentiel, diminuant ainsi l'impact des réformes sur les prix et autres domaines de l'autonomie décisionnelle des firmes.

Ensuite, parallèlement à la gestion des ressources humaines dans les entreprises, le personnel travaillant pour la $R \& D$ publique est très largement sous-utilisé ${ }^{2}$. Malgré une réhabilitation du rôle des intellectuels dans la société chinoise, ceux-ci sont loin de recevoir des stimulants à la hauteur des objectifs fixés par le gouvernement en matière de contribution au progrès technologique : faible réévaluation des salaires par rapport aux travailleurs manuels, rigidités dans la mobilité professionnelle, absence de protection de la propriété intellectuelle, évolution réduite des responsabilités professionnelles après une période de formation à l'étranger contribuent entre autres à une faible motivation. De ce fait, le nombre d'étudiants en troisième cycle dans les matières liées à l'électronique stagne dans un pays qui compte déjà un des plus faibles taux au monde d'étu-

1. $80 \%$ des firmes de notre échantillon ne subissaient pratiquement aucune pression concurrentielle.

2. Une enquête nationale menée en 1988 révélait que seulement $16 \%$ d'un échantillon de 34000 employés dans le domaine de la R\&D publique déclaraient pouvoir utiliser pleinement leurs capacités (R. Conroy, 1992). 
diants en sciences (18\% habitants), augurant à moyen terme de graves problèmes de renouvellement des enseignants et du personnel qualifié.

Enfin, les instituts de recherche nationaux, qui concentrent une bonne partie des capacités de R\&D, conservent une offre encore globalement inadaptée par rapport aux besoins des utilisateurs. Environ $75 \%$ des résultats de ces institutions restent à l'état d'échantillons ou de prototypes sans trouver d'acheteurs. Parallèlement, la diffusion des résultats à l'ensemble du tissu industriel est très réduite : sur 144 résultats transférés aux unités de production, $60 \%$ ont été utilisés par une seule entreprise, $17 \%$ par deux entreprises, $16 \%$ par plus de cinq entreprises ( $R$. Conroy, 1992). Les grandes entreprises d'Etat restent relativement peu demandeuses de technologie sur le plan intérieur. Le montant des sommes consacrées à l'achat d'équipements étrangers est d'ailleurs cinq fois plus élevé que le commerce intérieur de technologie. Un tel décalage indique une participation très limitée des instituts de recherche nationaux aux tentatives de modernisation des entreprises'. Il convient de signaler également qu'un des handicaps majeurs dans le faible développement du marché de la technologie, et plus spécifiquement de l'industrie du logiciel, réside dans l'absence d'un cadre juridique de protection de la propriété intellectuelle, la loi sur les droits d'auteurs n'ayant été promulguée que le $1^{\text {er }}$ juin 1991 . On notera enfín la dissémination des faibles ressources publiques destinées à l'innovation. De multiples recherches parallèles disposant de fonds peu importants sont menées par différentes institutions sans aucune coordination. L'industrie chinoise des circuits intégrés fournit un bon exemple de ce phénomène : les structures industrielles sont disséminées en un grand nombre d'entités ministérielles : ministère de l'Electronique, ministère des Télécommunications, ministère de l'Aéronautique. Il aurait été consacré à la R\&D durant le VI ${ }^{c}$ Plan (1981-1985) seulement 170 millions de dollars us, soit $0,6 \%$ en moyenne du chiffre d'affaires annuel des entreprises du secteur ou bien $100000 \$$ par entreprise, ce qui apparaît dérisoire au regard des sommes actuellement allouées par les entreprises des pays industrialisés.

\section{CONCLUSION}

Au total, la pérennisation d'une régulation bureaucratique des entreprises d'Etat a entraîné une série de distorsions dans la conduite du processus de transfert et empêché une accumulation optimale de capacités technologiques. L'intervention massive de l'Etat chinois s'est effectuée sans une refonte de ses moyens d'action. La modernisation a été plutôt décrétée à coup de décisions administratives sans toutefois briser le réseau de dépendances bureaucratiques dans lequel sont insérées les entreprises d'Etat. L'héritage industriel et techno-

1. R. Conroy indique que sur 620 entreprises ayant introduit des technologies étrangères, seules $2 \%$ avaient collaboré avec des instituts de recherche. 
logique de la Chine depuis la fin du siècle dernier compte pour beaucoup. D'une part la Chine ne bénéficie pas d'une longue période d' "incubation " de modernisation industrielle comme pour le Japon depuis l'époque Tokugawa et d'une modernisation de l'Etat depuis l'époque du Meiji. D'autre part, à la différence des NPI asiatiques, la Chine supporte toujours le poids de la stratégie industrielle suivie depuis 1949. Le cas de la province du Guandong qui, jusqu'en 1978, était très faiblement industrialisée, est sensiblement différent de celui des vieilles régions industrielles comme Shanghaï ou le Dongbei. La province du Guandong a construit du nouveau, à l'abri des statuts spéciaux en matière d'ouverture économique que lui a conféré le pouvoir central en 1978 et a pu profiter de l'apport financier et organisationnel de Hongkong : en ce sens l'industrialisation rapide depuis 1978 a été facilitée par l'absence de coûts importants inhérents à la transition d'un système industriel socialiste, et s'apparente de ce fait à la situation des NPI de la deuxième vague (Thaïlande, Malaisie, Indonésie). Les cas de Shanghaï et du Dongbei sont différents; même si ces deux provinces sont en mesure de bénéficier d'un apport de capitaux étrangers, elles devront supporter le poids de la transition d'une industrialisation de type stalinienne. La dégradation structurelle de la situation économique des entreprises d'Etat depuis 1988 est là pour le rappeler.

Aussi certaines politiques en matière de rattrapage technologique copiées sur les vertueux voisins asiatiques ont été le plus souvent considérées et utilisées sans tenir compte de leur enracinement historique et national fortement spécifique. Le processus, qui mène un territoire de l'importation de technologies étrangères à une accumulation efficace de capacités technologiques dans le cadre d'une industrie globale, comporte des éléments idiosyncratiques liés au contexte spatio-temporel différent dans lequel se déroulent les tentatives nationales de rattrapage technologique. En effet, chaque appareil industriel national est inséré dans un " milieu " politique, institutionnel, culturel spécifique; il dispose en outre d'un passé technico-industriel qui lui est propre et qu'il faut gérer, adapter et surtout faire évoluer aux regard d'objectifs qui se définissent par rapport au fonctionnement mondial des industries. En quelque sorte, il s'agit, pour la nation concernée, de la recherche d'une cohérence évolutive entre les possibilités d'adaptation et d'ajustement de son appareil de production avec les occasions d'accumulation de capacités technologiques laissées par un fonctionnement de type mondial de l'industrie électronique. Dans ce contexte, le transfert de technologie par l'IDE s'avère beaucoup plus efficace. Il permet aux firmes d'échapper au moins en partie au mode de régulation bureaucratique existant dans le secteur d'Etat, relâchant ainsi la plupart des contraintes systémiques qui bloquent le transfert de technologie dans les entreprises d'Etat ; mais l'IDE permet surtout le transfert de composantes (souvent tacites) organisationnelles, managériales des technologies, connaissances qui font terriblement défaut dans le contexte de transition d'une économie socialiste et sur lesquelles se fondent de plus en plus l'efficacité des équipements. 
L'industrie électronique chinoise reste encore très dépendante des transferts de technologies. Les priorités inscrites dans le VIII ${ }^{c}$ Plan semblent constituer la preuve d'une évolution à la fois irréversible sur le plan de l'ouverture aux technologies étrangères ainsi que d'une réorientation de la stratégie d'acquisition axée sur la coopération technologique et industrielle (IDE inclus). La diversification des pays investisseurs au début des années 1990, notamment les EtatsUnis, l'Europe et surtout le Japon, ainsi que le développement très rapide des filiales contrôlées à $100 \%$ devraient garantir un apport technologique plus conséquent que celui véhiculé dans les années 1980 par les investissements de Hongkong. Quant aux entreprises d'Etat, malgré un recours accru à la coopération technologique et industrielle étrangère, leur accumulation de capacités technologiques reste soumise en dernière instance aux succès des réformes dans le fonctionnement du secteur d'Etat, réformes qui touchent aussi bien le contrôle de la propriété que le système fiscal et bancaire et dont les issues débordent très largement le seul cadre économique.

BIBLIOGRAPHIE

Chang Dakai, Qiye touzi jizhi gaige de lilun yu zhengce (Politique et théorie de la réforme du système d'investissement des entreprises), in Xinjiang Caijing, ${ }^{\circ} 3,1989$, p. 35-38.

Chang Weimin, Woguo gongye qiye jingji guimo jiegou de shizheng fenyxi (Analyse de la structure des économies d'échelle dans l'industrie chinoise), in Zhonguo Gonye Jingji Yanjiu, 1991, n $^{\circ}$ 5, p. 43-48.

Conroy R., L'évolution technologique en Chine, Paris, OCDE, 1992.

Dalhman C. J., Ross-Larson B. et Westphal L. E., Managing Technological Development : Lessons from the Newly Industrializing Countries, in World Development, vol. $15, \mathrm{n}^{\circ} 6,1987$, p. $759-777$.

Fruin M., The Japanese Enterprise System, Oxford, Clarendon Press, 1994.

Hanson P. et Pavitt K., The Comparative Economics of Research Development and Innovation in East and West : A survey, New York, Harwood, Academic Publishers, 1987.

Huchet J.-F., Les transferts de technologies des entreprises japonaises dans l'industrie électronique chinoise, in Ebisu, $n^{\circ} 8$, janvier-mars 1995, Maison franco-japonaise, Tokyo.

Huchet J.-F., Transfert de technologie et industrialisation tardive. Le cas de l'industrie électronique en République populaire de Chine, 1978-1991, thèse de doctorat de sciences économiques, Université de Rennes I, 1993, 691 p.

Judet P., Les transferts de technologie, expériences dans les PVD et NPI asiatiques, in Revue Tiers Monde, t. XXX, no 120, octobre-décembre 1989, p. 777-796.

Kang Chen, The Failure of Recentralization in China : Interplays among Entreprises, Local Governments and the Center, Washington, DC, World Bank, Research Paper, 1990.

Korzec M., Labour and the Failure of Reform in China, New York, St. Martin Press, 1992.

Lall S., Promouvoir la compétitivité industrielle dans les pays en développement, Paris, OCDE, 1990.

Rehn D. et Simon D. F., Technological Innovation in China. The case of the Shanghai Semiconductor Industry, Cambridge, Ballinger Publishing Company, 1988. 
Simon D. F., China's Acquisition and Assimilation of Foreign Technology : Beijing's Search for Excellence, in Joint Economic Commitee (ed.), China's Economic Dilemmas: : The Problem of Reforms, Modernisation and Interdependence, vol. II, Washington, DC, Congress of United States, 1991.

Simon D. F. and Goldman M., Science and Technology in Post-Mao China, Cambridge, Harvard University Press, 1989.

Winiecki J., The Distorted World of Soviet Type Economies, Londres, Routledge, 1988.

Zhang Guoying, Dui woguo jishu yinjin jingji xiaoyi de sikao (Réflexions sur les profits retirés de l'introduction des technologies et équipements étrangers), in Jingji Congheng, $\mathrm{n}^{\circ}$ 9, 1989, p. 14-17. 